\title{
Infarto de la arteria de Percheron: reporte de un caso y revisión de la literatura
}

\section{Artery of Percheron Infarction: A Case Report and Review of the Literature}

\author{
H. Muñoz Quirland ${ }^{1} \quad$ V. Lázaro González ${ }^{1}$ \\ ${ }^{1}$ Centro Hospitalario de Pontevedra, Pontevedra, España \\ Rev Argent Radiol 2018;82:184-186.
}

Estimados editores,

El accidente cerebro vascular es una patología frecuente, que cuando ocurre en un territorio dependiente de la circulación posterior del cerebro, representa un reto diagnóstico por su variada presentación clínica. En ocasiones, puede simular otros procesos patológicos como la hemorragia intracraneal, infección y patología inflamatoria, entre otros. ${ }^{1}$

El tálamo y mesencéfalo presentan una irrigación sanguínea compleja que proviene de ramas perforantes de la arteria comunicante posterior (AcomP) y el primer (P1) y segundo segmento (P2) de la arteria cerebral posterior (ACP). La vascularización del tálamo suele clasificarse en 4 territorios. El territorio anterior es suplido por las arterias tálamotuberales que se originan de la AcomP. El territorio paramedial es irrigado por las arterias paramedianas que surgen del segmento P1 de la ACP, y los territorios inferolateral y posterior por las arterias tálamogeniculadas y coroideas posteriores respectivamente, que provienen del segmento P2 de la ACP.,3

Existe una variante anatómica atípica de las arterias paramedianas llamada arteria de Percheron, descripta en 1973 por el neurólogo francés Gerard Percheron, caracterizada por ser una arteria única y dominante que se origina del segmento P1 de la ACP e irriga el territorio paramedial bilateral talámico y parte del mesencéfalo anterior. La oclusión de dicha arteria provoca infarto talámico paramedial bilateral sin o con afectación mesencefálica. ${ }^{4,5}$

Comunicamos el caso de una paciente mujer de 49 años con factores de riesgo cardiovascular, que fue llevada al servicio de urgencias por presentar mareos y alteración súbita de la conciencia. Las pruebas complementarias de rutina fueron normales. Se realizó una tomografía computada (TC) cerebral sin contraste en donde no se

received

July 19, 2017

accepted

February 27, 2018

published online

April 27, 2018
Address for correspondence $H$. Muñoz Quirland, Centro Hospitalario de Pontevedra, Mourente s/n, CP:36161, Pontevedra, España (e-mail: hugo.munoz83@gmail.com).

evidenciaron colecciones hemáticas intracraneanas ni signos de isquemia. Posteriormente, la paciente evolucionó con mayor deterioro de la conciencia, por lo que fue trasladada a la unidad de cuidados intensivos (UCI) y se realizó una angiotomografía cerebral que no demostró defectos de repleción vasculares ni lesiones nuevas. Ante ese contexto, se solicitó una resonancia magnética (RM), cerebral y una angioresonancia intracraneana sin gadolinio que mostró un área de hiperintensidad en secuencias potenciadas en T2, FLAIR, difusión con caída en el mapa de coeficiente de difusión aparente (ADC) e hipointensidad en secuencias ponderadas en $\mathrm{T} 1$ a nivel de la región paramedial de ambos tálamos; de configuración simétrica, y con compromiso del mesencéfalo superior (-Fig. 1). La secuencia 3D-TOF intracraneana mostró indemnidad de grandes vasos arteriales sin delimitarse oclusiones venosas profundas (-Fig. 2).

Los hallazgos se interpretaron como infarto isquémico subagudo precoz talámico-mesencefálico secundario a oclusión de la arteria de Percheron. La paciente se manejó conservadoramente y evolucionó de forma favorable, aunque no se demostró el origen cardioembólico, ya que la ecografía Doppler de troncos supraórticos y ecocardiograma transtorácico no evidenciaron alteraciones.

La incidencia estimada del infarto de la arteria de Percheron se sitúa alrededor del $0,1 \%$ al $2 \%$ de todos los accidentes cerebrovasculares y del $4 \%$ al $18 \%$ de los infartos talámicos. ${ }^{2}$ El infarto de la arteria de Percheron es una causa poco frecuente de coma en pacientes de mediana y tercera edad. Ambos sexos están afectados en la misma proporción y la mayoría presentan factores de riesgo cardiovascular. El infarto cardioembólico es la causa más frecuente, y algunas causas son la fibrilación auricular, aneurismas de la pared

Copyright @ 2019, Sociedad Argentina de Radiología. Publicado por Thieme Revinter Publicações Ltda., Rio de Janeiro, Brazil. Todos los derechos reservados.

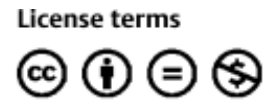

DOI https://doi.org/ $10.1055 / \mathrm{s}-0038-1642599$. ISSN 1852-9992. 

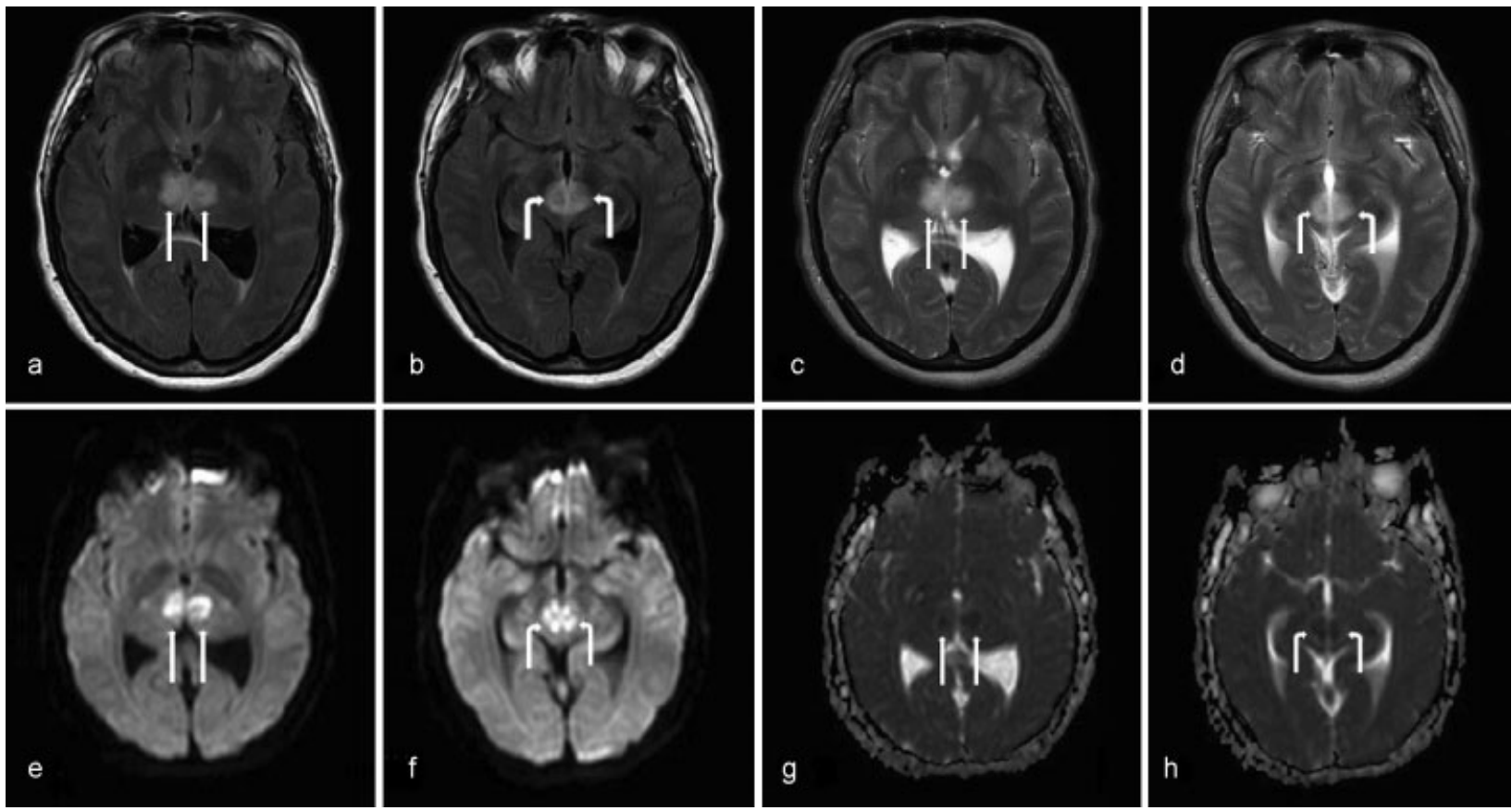

Fig. 1 Resonancia magnética (RM) en secuencias FLAIR (a,b), potenciadas en T2 (c,d), difusión (e,f) y coeficiente de difusión aparente (ADC) $(\mathrm{g}, \mathrm{h})$ en plano axial. Se observa el aumento de señal de configuración bilateral y simétrica en la región paramedial de ambos tálamos (flechas rectas) y mesencéfalo superior (flechas curvas). La intensidad de señal es normal del parénquima correspondiente a los ramos distales de ambas arterias cerebrales posteriores.

ventricular, cortocircuitos de derecha a izquierda y estados de hipercoagulabilidad. ${ }^{6}$

Existen cuatro variantes anatómicas de las arterias paramedianas que irrigan el tálamo (-Fig. 3): Tipo I: arterias paramedianas que surgen en cada ACP. Es la variante más frecuente. Variante IIa: arterias paramedianas que surgen independientes de una de las ACPs. Variante IIb: existencia de un tronco común (arteria de Percheron), que nace del segmento P1 de una de las ACPs. Variante III: las arterias paramedianas nacen de una arteria en forma de "arco" que comunica ambos segmentos P1 de la ACP. ${ }^{1,2,7}$

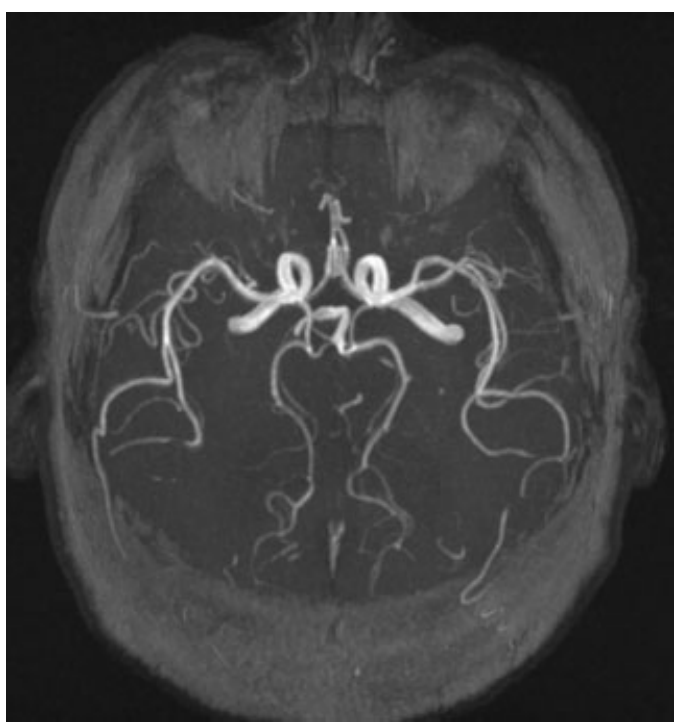

Fig. 2 Angioresonancia intracraneana con reconstrucción en proyección de máxima intensidad (MIP), en el que no se observan defectos de repleción en los grandes vasos arteriales intracraneales.
La TC puede mostrar hipodensidad en ambos tálamos y/o mesencéfalo medial o ser normal. En RM existe hiperintensidad en dichas localizaciones en secuencias potenciadas en T2, FLAIR y difusión, aunque dependen del estado evolutivo del infarto.
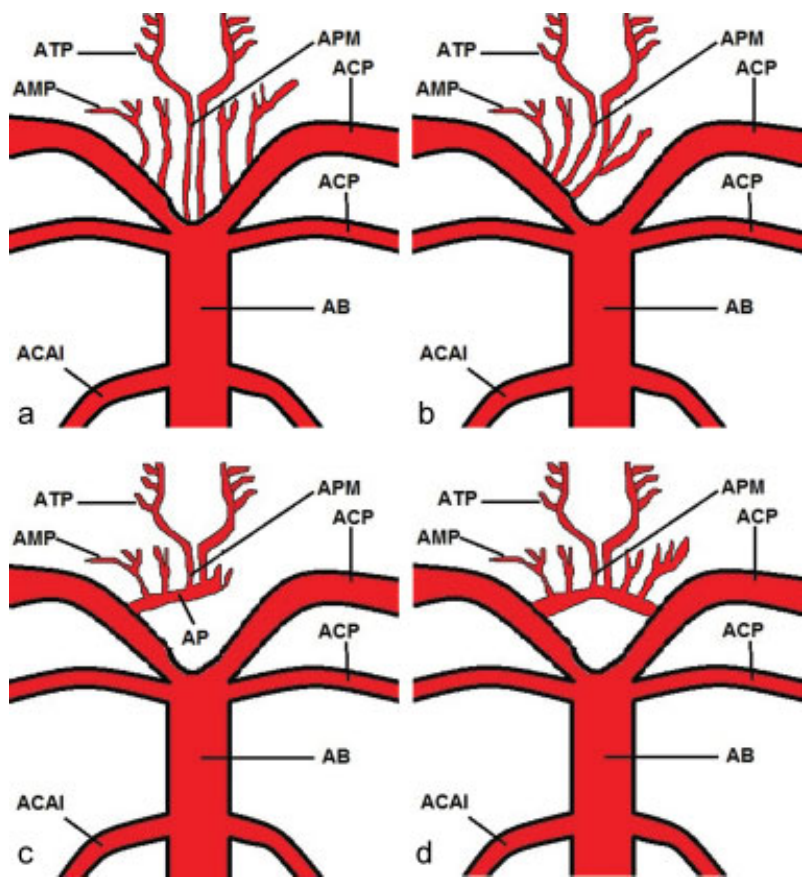

Fig. 3 Variantes anatómicas de la vascularización del tálamo y mesencéfalo. Tipo I (a), tipo Ila (b), tipo IIb (c), y tipo III (d). Abreviaturas: Arterias tálamo perforantes (ATP), arterias mesencéfalo perforantes (AMP), arterias paramedianas (APM), arteria cerebral posterior (ACP), arteria cerebelosa superior (ACS), arteria basilar $(A B)$, arteria cerebelosa anteroinferior (ACAI) y arteria de Percheron (AP). 
Otro hallazgo en RM es el signo de la "V" que consiste en una hiperintensidad en secuencia FLAIR y en difusión, en la superficie pial de la cisterna interpeduncular del mesencéfalo (sensibilidad del 67\%). Los protocolos angiográficos no invasivos deben realizarse para valorar la indemnidad del polígono de Willis y descartar una oclusión de la arteria basilar o del sistema venoso profundo. No obstante, no suele verse la arteria de Percheron por su fino calibre u oclusión proximal, pero se interpreta su compromiso por la localización característica del territorio talámico, que no podría presentarse con las otras variantes vasculares. La angiografía no siempre es necesaria para el diagnóstico y resulta útil cuando los métodos anteriores no son concluyentes, o bien, para fines terapéuticos específicos. ${ }^{2,4-6,8}$ Otros diagnósticos diferenciales del infarto de la arteria de Percheron son: encefalopatía de Wernicke, mielinolisis extrapontina, encefalitis viral, enfermedad de Wilson, gliomas, enfermedad de Creutzfeldt-Jakob, aunque las lesiones no siguen un territorio vascular conocido. ${ }^{6,8}$

El tratamiento suele ser de soporte, idealmente en una unidad de cuidados intensivos, ya que los pacientes presentan menor morbimortalidad. Es importante avanzar rápidamente en el algoritmo diagnóstico en el contexto de un paciente con trastorno de conciencia severo sin hallazgos tomográficos que lo justifiquen. En general los infartos talámicos bilaterales tienen un pronóstico favorable, pero en algunos casos pueden representar un verdadero desafío. ${ }^{6}$

\section{Confidencialidad de los Datos}

El autor declara que ha seguido los protocolos de su centro de trabajo sobre la publicación de datos de pacientes y que todos los pacientes incluidos en el estudio han recibido información suficiente $\mathrm{y}$ han dado su consentimiento informado por escrito para participar en dicho estudio.

\section{Responsabilidades Éticas}

Protección de personas y animales. Los autores declaran que para esta investigación no se han realizado experimentos en seres humanos ni en animales.
Confidencialidad de los datos. Los autores declaran que en este artículo no aparecen datos de pacientes.

Derecho a la privacidad y consentimiento informado. Los autores declaran que en este artículo no aparecen datos de pacientes.

Nota

El trabajo se realizó en el Complejo Hospitalario de Pontevedra con dirección Mourente s/n, CP: 36161, Pontevedra, España.

Conflicto de Intereses

El autor del trabajo declara no tener ningún conflicto de intereses. No hay fuente de financiación.

\section{Bibliografía}

1 Anderson C, O'Brien R. Occlusion of the artery of Percheron: an unusual cause of bilateral stroke. BMJ Case Rep 2012; 19

2 Lamot U, Ribaric I, Popovic KS. Artery of Percheron infarction: review of literature with a case report. Radiol Oncol 2015;49(02): 141-146

3 Lazzaro NA, Wright B, Castillo M, et al. Artery of percheron infarction: imaging patterns and clinical spectrum. AJNR Am J Neuroradiol 2010;31(07):1283-1289

4 Zappella N, Merceron S, Nifle C, et al. Artery of Percheron infarction as an unusual cause of coma: three cases and literature review. Neurocrit Care 2014;20(03):494-501

5 Caruso P, Manganotti P, Moretti R. Complex neurological symptoms in bilateral thalamic stroke due to Percheron artery occlusion. Vasc Health Risk Manag 2016;13(13):11-14

6 Rodriguez EG, Lee JA. Bilateral thalamic infarcts due to occlusion of the Artery of Percheron and discussion of the differential diagnosis of bilateral thalamic lesions. J Radiol Case Rep 2013;7 (07):7-14

7 Turner J, Richardson T, Kane I, Vundavalli S. Decreased consciousness: bilateral thalamic infarction and its relation to the artery of Percheron. BMJ Case Rep 2014;2014:xx

8 Vinod KV, Kaaviya R, Arpita B. Artery of Percheron infarction. Ann Neurosci 2016;23(02):124-126 